\title{
Prediction and prevention of IDDM
}

\author{
M. H oneyman ${ }^{1}$, C. Wasserfall ${ }^{2}$ J J N erup $^{3}$, A . R ossini ${ }^{4}$ \\ ${ }^{1}$ The Walter and Eliza Hall Institute,Victoria, Australia \\ ${ }^{2}$ University of Florida, Gainesville, Florida, USA \\ ${ }^{3}$ Steno Diabetes Center, Gentofte, Denmark \\ ${ }^{4}$ University of Massachusetts, Massachusetts, USA
}

While much progress has been made in the treatment of insulin-dependent diabetes mellitus (IDDM), one in 200 children still die at clinical onset in some countries. There is thus an urgent need not only for greater public awareness of the symptoms of IDDM, but particularly for wide screening linked to intervention, ultimately leading to prevention of the disease. Recent natural history studies $[1,2]$ have demonstrated that the onset of clinical symptoms of IDDM is preceded by a period of asymptomatic autoimmunity. There has been a constant evolution in identifying markers of this state, which include antibodies and T-cell responses to islet autoantigens, predominantly islet cell antibodies (ICA), (pro)insulin (insulin autoantibodies, IAA), glutamic acid decarboxylase (GAD) and the tyrosine phosphatase, IA-2 (ICA512). Genetic markers include HLA risk alleles (HLA-DQ, DR and class I) [3] and non-HLA genes conferring risk are also being studied $[4,5]$. This has led to the emergence of strategies to intervene in the development of IDDM prior to the total loss of beta-cell mass. The first form of screening was by metabolic studies, involving measurement of first-phase insulin release (FPIR) following glucose challenge. However, this was cumbersome and invasive and importantly only available to small well-defined groups of those already suspected to be at risk, such as the population

Participants: G. Eisenbarth, Barbara Davis Center for Childhood Diabetes, Denver Colorado, USA

P. Bingley, Southmead Hospital, Bristol, UK

E. Gale, Southmead Hospital, Bristol, UK

N. Maclaren, University of Florida, Gainesville, Florida, USA L. Harrison, Walter and Eliza Hall Institute, Melbourne, Australia

P. Vardi, Tel Aviv University, Petah-Tivka, Israel

G. Klingensmith, Barbara Davis Center for Childhood Diabetes, Denver, Colorado, USA

Corresponding author: M. C. Honeyman, The Walter and Eliza Hall Institute of Medical Research, Post Office, Royal Melbourne Hospital, Parkville, Victoria 3050, Australia of first-degree relatives of diabetic individuals ("relatives"). However, $90 \%$ of new cases of childhood diabetes are in individuals in the general population, in whom the risk is known to be 10-15 times lower than in first-degree relatives. The subsequent development of the ability to measure both genetic susceptibility by HLA class II antigens and immunologic humoral and cellular markers of islet autoimmunity has conferred the ability to screen a much wider population and to identify individuals at risk.

Large studies have therefore been undertaken in a number of different populations, involving both atrisk relatives and the general population in order to determine the most effective screening technique(s). The studies reported in this workshop cover screening of family/second generation members, schoolchildren and newborn cohorts, and the psychosocial impact of such screening upon families. Updates were also given on the trials which have commenced preventative therapies. Two major forms of risk assessment suitable for large-scale population screening were discussed.

\section{George Eisenbarth, Penelope Bingley: Genetic markers in risk assessment}

While the initial triggering event(s) in the development of islet cell autoimmunity remains unknown, the HLA molecules DQA1(*0301)-DQB1(*0302) ( = DQ8), DQA1 $(* 0501)-\mathrm{DQB} 1(* 0201) \quad(=\mathrm{DQ} 2)$, DRA-DRB1 $(* 0401) \quad(=\mathrm{DR} 4)$ and DRA-DRB1 $(* 0301)(=\mathrm{DR} 3)$ are known to confer susceptibility to IDDM. Screening for these molecules has been used as an entry point for the Diabetes Autoimmunity Intervention Study of the Young (DAISY) [6], where 50000 Colorado newborns will be tested for the presence of DR3 and DR4. Of the 12000 results to date, the genotype DR3,4 is present in $2.3 \%$ and DR4,X ( $\mathrm{X}=$ non-DR3, non-DR4), in approximately $20 \%$. These are present in $40 \%$ and $20 \%$ respectively of 
IDDM subjects [3] and therefore confer increased risk. However, $1 \%$ of IDDM subjects, compared to $20 \%$ of the general population express DQB1 $(* 0602)$ ( = DQ6). Thus, DQ6 may be used to indicate Caucasoid children at lower risk, and it was hypothesized by George Eisenbarth that diabetic but antibody negative children with DQ6 (most of whom are non-Caucasoid) may not in fact have IDDM, but a non-autoimmune form of diabetes. Even larger birth cohort studies are ongoing in Scandinavia and Finland. In the USA-wide Diabetes Prevention Trial-1 (DPT-1) of 44384 relatives, DQ6 has been used as an exclusion criterion subsequent to autoantibody testing. Dr. Eisenbarth reported that to date, of the 52 ICA-positive, DQ6 positive subjects in the DPT-1, 20\% had unconfirmed ICA, while of the remainder, $90 \%$ of those less than 10 years of age had ICA but not GAD, IAA or IA-2 antibodies. Of the 11-20 year olds, most individuals had one or two but not three antibodies, leading to the conclusion that DQ6 is associated with less and later development of autoantibodies. Caution was further stressed by Polly Bingley in the use of genetic markers alone to indicate risk. She discussed the use of Relative Risk and Absolute Risk, demonstrating that while Relative Risks for a marker could be very high, when populations are considered, the Absolute Risk ((marker prevalence in the test population/ marker prevalence in the background population) $\times$ overall risk) must be used. This may result in an unacceptably low predictive capacity. She cited an example from the Belgian National Diabetes Registry, where even in the youngest age group (0-9 years), the highest risk genotype (DQ2/DQ8) was present in $52 \%$ of IDDM cases, but only $2 \%$ of the control subjects. This gives a Relative Risk of 69.6, but an Absolute Risk of only $5 \%$. Therefore, new markers or combinations need to be identified which achieve greater discrimination.

Dr. Bingley further pointed out that insufficient attention has been paid to the evaluation of the Absolute Risk associated with genetic markers, and many studies are still not population-based. In addition, even in the largest population-based studies, the very wide $95 \%$ confidence intervals around the Absolute Risk are not given. Since stratification according to age of diagnosis is also necessary in population studies, such analyses demand very large numbers of cases to achieve reasonable confidence intervals in the subgroups. The size of the control population often receives insufficient consideration, but is usually the major determinant of the confidence interval. The use of more specific marker combinations, such as autoantibodies and T-cell responses, as well as HLA and non-HLA susceptibility alleles, means that very large samples of the background population will be required for future studies.

Len Harrison added that typing for HLA-class I should not be overlooked, as HLA-A24 had been shown to indicate high risk for rapid onset in at-risk relatives [3] in some Caucasoid and Asian populations.

\section{George Eisenbarth, Penelope Bingley, Noel Maclaren:} Immunity-based risk assessment

Autoantibody-based risk assessment differs from genetic assessment of risk, as the variables are continuous rather than discrete, and may vary over time. In addition, sample-related spurious signals, for example due to interfering antibodies or high IgG levels, are likely to represent a greater problem. Dr. Eisenbarth reported that in studies in relatives and the DAISY cohort [1, 6], IAA prevalence dropped with increasing age of onset of IDDM, but that GAD and IA-2 antibodies remained stable over time from age 3 years once they appeared. An increasing number of autoantigens were recognized by antibodies over time, with different combinations of IAA, GAD and IA-2 appearing prior to onset. Risk conferred by each antibody was additive, as no antibody indicated no or very low risk, one antibody low risk, two antibodies moderate risk and three antibodies high risk.

Dr. Bingley, in the Oxford study [7], compared 300 children with IDDM with 3000 schoolchildren of the same age. The results of this study reiterated that single markers cannot achieve the level of discrimination required if they are to be applied in the general population. Levels of multiple antibodies were elevated in more than $90 \%$ of the children at time of diagnosis, but in less than $1 \%$ of the schoolchildren. In $90 \%$ of the schoolchildren no antibodies were found, $8.7 \%$ had one, $0.5 \%$ had two, $0.1 \%$ had three and $0.14 \%$ had four (ICA, IAA, GAD, IA-2 antibodies). Thus, multiple antibodies appeared to be more discriminatory. Dr. Bingley suggested that the most effective risk assessment strategy would be a combination of genetic screening of newborns followed by autoantibody testing of those with HLA susceptibility genotypes. If the genetic screening indicated high early risk, autoantibody tests were suggested annually, if the risk was of later development, autoantibody testing at 5 and 10 years of age, and if lower risk was indicated, autoantibody testing at age 10 years. Alternatively, she suggested a high sensitivity autoantibody screening, simple and inexpensive to perform, of high potential throughput, e.g. for IA-2 or GAD, followed in positive subjects by a further test also of high sensitivity, but which could be more complex e.g. ICA or IAA.

Noel Maclaren added that studies in schoolchildren and relatives [8] showed that ICA in the absence of antibodies to insulin, GAD, IA-2 and IA-2 beta, conferred no or very low risk. However, the presence of any of these antibodies in addition to ICA conferred a risk for IDDM, which increased with the number of autoantibodies. All these studies taken together indicate 
that ICA need not be used as an initial screening test, and that IA-2 and/or GAD antibodies may suffice.

As the destruction of beta cells leading to IDDM is a T-cell-mediated process, T-cell assays are required as additional markers of risk. However, the recent workshop, the results of which were reported at the 1996 Immunology of Diabetes Society Meeting (Canberra, Australia), indicated that such assays are difficult to reproduce, with few exceptions [2] have not included HLA-matched control subjects, and are not yet well standardized throughout the world. Further workshops are therefore required to develop and trial and standardize new T-cell assays.

Edwin Gale, Noel Maclaren, Leonard Harrison: Prevention trials

The most recent data from two major prevention trials, the DPT-1 and the European Nicotinamide Diabetes Intevention Trial (ENDIT) were presented by Noel Maclaren and Edwin Gale. The DPT-1 trial is to test whether antigen-based immune interventions, shown to be effective in rodent models, can delay the onset of IDDM. Recombinant whole human insulin is given parenterally (twice daily subcutaneously as ultra-lente at $0.25 \mathrm{IU} \cdot \mathrm{kg}^{-1} \cdot \mathrm{day}^{-1}$ and yearly by infusion as regular insulin for 4 days), or orally as encapsulated regular insulin at $7.5 \mathrm{mg} /$ day. The parenteral therapy is given to high risk relatives (risk $>50 \%$ by 5 years) in a non-placebo-based randomized trial, and the oral therapy to relatives with an intermediate risk (risk $25-50 \%$ by 5 years) in a placebo-controlled trial. The parenteral arm began in December 1994 and the oral arm September 1996. This United States multi-centre trial so far involves 10 centres, 144 affiliates and 210 satellites. To date 44384 relatives have been screened for ICA and $3.4 \%$ found positive with higher frequencies in males $(4.1 \%)$ than females $(2.9 \%)$. Of 1414 eligible, $68.7 \%$ have been staged $(n=971)$, and $271(28 \%$ of the IVGTTs done, or $19 \%$ of those found to be ICA + ) of those staged were found to be at high risk (no HLA-DQB1*0602/3 and first phase insulin release measured by $1+3$ min plasma insulin levels after $0.5 \mathrm{~g}$ i.v. glucose $<10$ th percentile). To date 157 ICA-positive relatives (11.1\%) developed IDDM before randomization. The DPT-1 was designed to screen 60000 relatives at 15000 per annum for 4 years yielding 540/year for staging at an ICA-positive frequency of $3.6 \%$. The sensitivity of the trial was to test the rescue of a third of those treated with insulin in the parenteral trial, and one half of those given oral insulin. Of the 540 expected, $35 \%$ should go to the high risk group (180/year) with half expected to agree to consent, whereas $40 \%$ (215/year) would go to the intermediate risk group with two thirds expected to consent. To date 148 relatives have been randomized to the parenteral insulin arm $(80 \%$ of target) and 46 to the oral arm (98\% of target).

Dr. Gale reported that the aim of ENDIT is to test the null hypothesis that high dose oral nicotinamide $\left(1.3 \mathrm{~g} / \mathrm{m}^{2}\right)$ cannot achieve a $35 \%$ reduction in incidence of IDDM in ICA-positive, first-degree relatives. This trial is a prospective, placebo-controlled double-blind study over 5 years of treatment. The target number of subjects is 422 , i.e. 211 in each arm. The subjects enrolled are first-degree relatives, aged 5-40 years, of an individual who developed IDDM under the age of 20 years, and who are themselves positive for ICA $\geq 20 \mathrm{JDF}$ units on repeated testing. Twenty national groups are participating, the major players being Canada, Finland, Poland, UK, Denmark, Sweden and Norway. Progress to date: more than 35000 first-degree relatives have been screened, with 481 randomized to treatment from a total of 528 . This allows for a drop-out rate of $20 \%$. The projected completion date is June 1997. To date, 34 subjects have progressed to IDDM and 39 have dropped out, which remains within estimates. Almost all dropouts were within the first 6 months, the main reasons being difficulty with swallowing tablets (children), or non-compliance. Blinded comparisons are performed at 6-month intervals by a review committee, and at the latest review (February 1997) no problems had been reported to the investigators. An interim statistical review will be carried out in September 1998 and the study will end in approximately June 2002.

Len Harrison reported that a double-blind, placebo-controlled, crossover designed intra-nasal insulin trial had commenced in Melbourne, Australia in June 1996. To date 26 subjects have started treatment, and are being tested monthly for autoantibodies (IAA, GAD, IA-2) and T-cell proliferative and cytokine (interferon-gamma, interleukin (IL)-10, IL-5, tumour necrosis factor-alpha) responses to relevant antigens. The study will be completed at the end of 1998.

There was discussion on how to fund future largescale trials of this nature. The suggestion was put forward that the investigative community approach the National Institutes for Diabetic, Digestive and Kidney Disease (NIDDK) and Allergy and Infections Diseases Natinal Institue (NIAID) to fund jointly future large-scale international screening studies which may extend beyond 5 years. Such studies could also include funding from other sources in countries other than the United States. This form of funding could be used by NIH as a model for future collaborative studies in an era of shrinking governmental funding.

\section{Pnina Vardi, Georgeanna Klingensmith: Psychosocial impact of diabetes prediction}

Diagnosis of IDDM is accompanied by varying degrees of psychosocial disturbances, and as most of 
the patients are young, such disturbances engulf both the diabetic child and their family. However there is little information on how affected individuals and their families respond to prediction of IDDM. Pnina Vardi reported on a pilot study with Avinoam Galazer, in which autoantibody positive individuals were identified through a large-scale screening programme for ICA. Nine families with 10 autoantibody youngsters were identified. Eight IDDM and 9 healthy siblings were included as control groups. The reaction of a group of 15 parents was examined at the time of diagnosis of clinical IDDM. The response to the detection of autoantibody positivity was assessed within a week of the notification of parents and again after 3 months by a structured questionnaire and with the Impact of Events Scale (IES) [10], which assesses intrusion (intrusively experienced ideas, images, feelings or bad dreams) and avoidance (consciously recognized avoidance of unpleasant ideas, feelings or situations). In parents (mothers more than fathers), intrusion and avoidance were as high at notification, as in the parents at diagnosis, but a few months later the scores had dropped significantly. For the ICA-positive child and the IDDM sibling, intrusion and avoidance were low at both time points. As stress was greatest at the time of diagnosis, it was recommended that the offer of screening would be best left until several months post diagnosis.

Georgeanna Klingensmith reported that most of our current knowledge comes from screening relatives. Similarly to Dr. Vardi, she reported that family members showed increased anxiety (State-Trait Anxiety Inventory Test) within a week of learning their antibody-positive status. When tested again at 2-4 months after notification, the anxiety scores had returned to normal. The Colorado group has also begun to evaluate the psychosocial impact of IDDM prediction in their DAISY study. In particular, a child vulnerability questionnaire was used to determine whether participants in the DAISY project were perceived as being more vulnerable than the normative sample. The data so far suggest that younger children participating in a diabetes risk identification study are perceived to be more vulnerable than older children, although it was noted that this could be an artefact of the vulnerability scale, and validation is necessary. Klingensmith further noted that vulnerability was not altered by antibody status, degree of genetic risk identified or presence of a family member with IDDM. Therefore, to date, IDDM prediction does not appear to cause psychosocial harm, however further studies are required to evaluate this important aspect of IDDM prediction particularly if intervention trials with young children in the general population are to be undertaken. The availability of support and counselling are an important part of any disease risk screening programme.

Investigators should be aware of the psychosocial aspects of screening studies, and therefore include parents of children with and without IDDM, and individuals with IDDM, in committees to plan screening protocols.

Screening for risk markers would be best offered to family members, preferably not at the time of diagnosis of the proband, once the stress of the diagnosis has diminished. Screening should only be offered in the context of research studies into prevention or natural history of the disease.

\section{Future directions and recommendations}

- Intervention strategies, currently being tested in pilot studies, may be capable of delaying the clinical onset of IDDM perhaps indefinitely. Co-operative networks, which include experienced investigator groups, are now required to co-ordinate large multicentre intervention studies.

- The planning phase of intervention studies should include representatives from patient advocacy groups. - Additional immunological and genetic markers, including standardized T-cell and other assays, are required. These are to identify the pre-diabetic state, to monitor the progression of the disease in order to determine the appropriate timing of intervention, and to monitor the efficacy of appropriate intervention modalities.

\section{References}

1. Verge CF, Gianani R, Kawasaki E et al. (1996) Prediction of type 1 diabetes in first-degree relatives using a combination of insulin, GAD, and ICA512/IA-2 autoantibodies. Diabetes 45: 926-933

2. Honeyman MC, Stone N, de Aizpurua H, Rowley M, Harrison LC (1997) High T-cell responses to the glutamic acid decarboxylase (GAD) isoform 67 reflect a hyperimmune state that precedes the onset of IDDM. J Autoimmun 10: 165-173

3. Honeyman MC, Harrison LC, Drummond B, Colman PC, Tait BD (1995) Analysis of families at risk for IDDM reveals that HLA antigens influence progression to clinical disease. Mol Med 1: 576-582

4. Wicker LS, Todd JA, Peterson LB (1995) Genetic control of autoimmune diabetes in the NOD mouse. Ann Rev Immunol 13: 179-200

5. Morahan G, Huang D, Tait BD, Colman PG, Harrison LC (1996) Markers on distal chromosome $2 \mathrm{q}$ linked to IDDM. Science 272: 1811-1813

6. Rewers M, Bugawan TL, Norris JM et al. (1996) Newborn screening for HLA markers associated with IDDM: diabetes autoimmunity study in the young (DAISY). Diabetologia 39: 807-812

7. Pinkey JH, Bingley PJ, Sawtell PA, Dunger DB, Gale EA (1994) Presentation and progress of childhood diabetes mellitus: a prospective population-based study. The Bart's-Oxford Study Group. Diabetologia 37: 70-74

8. Cantor AB, Krischer JP, Cuthbertson DD et al. (1995) Age and family relationship accentuate the risk of IDDM in relatives of patients with IDDM. J Clin Endocrinol Metab 80: 3739-3743

9. Gale EA (1996) Nicotinamide: potential for the prevention of type 1 diabetes? Hormone \& Metabolic Research 28: 361-364

10. Zilberg NJ, Weiss DS, Horowitz MJ (1982) Impact of event scale: a cross-validation study and some empirical evidence supporting a conceptual model of stress response syndromes. J Consult Clin Psych 50: 407-414 